\title{
ANÁLISIS DEL EFECTO DE LA FORMACIÓN B-LEARNING EN EL PROFESORADO. ESTUDIO DE CASO DE UNA COOPERATIVA DE ENSEÑANZA
}

\section{ANALYSIS OF THE EFFECT OF B-LEARNING TRAINING ON THE TEACHING STAFF. CASE STUDY OF A TEACHING COOPERATIVE}

\author{
Jesús López Belmonte \\ Universidad Internacional de Valencia, Espanha \\ jesus.lopezb@campusviu.es \\ Santiago Pozo Sánchez \\ Universidad de Granada, Espanha \\ santiagopozo@correo.ugr.es \\ Arturo Fuentes Cabrera \\ Universidad de Granada, Espanha \\ arturofuentes@ugr.es \\ Gerardo Gómez García \\ Universidad de Granada, Espanha \\ gerardoacci@correo.ugr.es
}

RESUMEN: La implementación de las TIC en los centros educativos es una realidad que afecta a muchos docentes que - desafortunadamente - presentan una competencia escasa en su manejo y su conocimiento. Es por ello que diferentes proyectos estatales y autonómicos se han centrado en desarrollar una formación en competencia digital hacia estos docentes con la finalidad de cambiar este panorama. El presente trabajo tiene como objeto conocer la incidencia del proyecto INNOVACOOP en el cuerpo docente de una cooperativa de enseñanza de la Ciudad Autónoma de Ceuta (España). El método del trabajo es experimental de tipo pretest y postest, en la que se ha aplicado entre ambos, un plan de formación blended learning sobre competencia digital. La medición de respuestas se llevó a cabo a través de la creación de un cuestionario ad hoc que trató las diferentes dimensiones que conforman la competencia digital, así como factores personales y motivacionales. Con las respuestas recabadas se realizó un análisis descriptivo-correlativo, en la que se pretendió con la utilización de la prueba t-student conocer diferencias significativas entre las medias de ambos test, así como diferencias significativas entre los tamaños de efecto a través de la $d$ de Cohen y la correlación biserial. Los resultados reflejaron una mejora considerable en las diferentes dimensiones de la competencia digital, así como en la receptividad y motivación de los docentes hacia las TIC y su utilización en el aula, quedando patente la importancia de seguir trabajando en la formación permanente sobre tecnología en profesores en ejercicio.

PALABRAS CLAVE: competencia digital; profesorado; blended learning.

ABSTRACT: The implementation of ICT in schools is a reality that affects many teachers, who - unfortunately - have little competence in their management and knowledge. That is 
why different state and autonomous projects have focused on developing a digital competence training for these teachers in order to change this landscape. The purpose of this paper is to know the impact of the INNOVACOOP project on the teaching staff of a teaching cooperative in Ciudad Autónoma de Ceuta (Spain). The work method is experimental pretest and posttest, in which a blended learning plan on digital competence has been applied between both. The measurement of responses was carried out through the creation of an ad hoc questionnaire that dealt with the different dimensions that make up the digital competence, as well as personal and motivational factors. With the collected answers, a descriptive-correlative analysis was carried out, in which it was intended with the use of the t-student test to know significant differences between the means of both tests, as well as significant differences between the effect sizes through the $d$ of Cohen and the biserial correlation. The results reflected a considerable improvement in the different dimensions of digital competence, as well as in the receptivity and motivation of teachers towards ICT and its use in the classroom, being clear the importance of continuing working in permanent training on technology in teachers in exercise.

KEYWORDS: digital competence; teachers; blended learning.

\section{Introducción}

La sociedad actual se encuentra sometida a un cambio continuo propiciado por las constantes innovaciones que se producen en el campo tecnológico (ARZOLA; LOYA; GONZÁLEZ, 2017). El panorama social se caracteriza por haber sufrido un proceso de transformación desde una era analógica hacia una digital, en la que la práctica totalidad de la población utiliza la tecnología durante su vida cotidiana para satisfacer sus necesidades básicas, para interactuar con el resto de la sociedad y para llevar a cabo actividades de ocio (STEFANI; ANDRÉS; OANES, 2014).

Este entorno cambiante no ha afectado por igual a todos los individuos que componen la sociedad. De todos ellos, el grupo generacional que mayor atracción ha demostrado por la utilización de la tecnología como elemento intrínseco a su actividad diaria ha sido - sin lugar a dudas - la población juvenil (AREA, 2015). Este grupo generacional se ha constituido como un compendio de auténticos nativos digitales, es decir, aquellos que - en contraste con los inmigrantes digitales - han vivido la incorporación de las tecnologías de la información y la comunicación (TIC) en la vida diaria como algo natural, propio e innato (SÁNCHEZ; CASTRO, 2013).

Las TIC han supuesto una auténtica revolución en la manera en la que se conciben los procesos instructivos y formativos (VIÑALS; CUENCA, 2016). Bajo este paradigma socioeducativo, resulta pertinente que en el ámbito de la educación se fomenten políticas de inclusión de la tecnología por parte de los centros educativos, de manera que cuenten con el equipamiento necesario para poder trabajar la competencia digital y que los procesos de enseñanza y aprendizaje puedan adaptarse a la realidad actual y a las necesidades del discente de hoy en día (MÉNDEZ; DELGADO, 2016).

La educación avanza - en resumidas cuentas - hacia un proceso de actualización de los espacios de aprendizaje (GONZÁLEZ, 2017), una transformación orientada hacia la utilización adecuada de la tecnología por parte del profesorado y de los discentes para que la inmersión en el mundo digital resulte significativa y eficaz (CABERO, 2017). 
Sin embargo, el esfuerzo realizado por parte de los centros educativos por incorporarse al mundo digital debe ser directamente proporcional al realizado por los docentes en activo para llevar a cabo metodologías didácticas imbricadas en el mundo tecnológico. Este tipo de propuestas didácticas parten del discente como principal generador del aprendizaje, gracias a la acción mediadora y orientadora del docente (POZUELO, 2014), que debe poner en práctica su liderazgo tecnológico para promover una actitud positiva hacia el uso de la tecnología (MACÍAS; CHUM; ARAY; RODRÍGUEZ, 2018) y que -de esta forma- pueda producirse una verdadera inclusión de las TIC en los espacios de aprendizaje (RODRÍGUEZ; PEDRAJA, 2017).

Afortunadamente, buena parte del colectivo docente ha contemplado en las TIC una oportunidad para dinamizar y actualizar los procesos instructivos (MURILLO; KRICHESKY, 2015), considerando imprescindible la inclusión de dispositivos electrónicos y entornos digitales en sus metodologías didácticas (MCGILL; KOPPI; ARMAREGO, 2014; VACEK; RYBENSKA, 2015; MURILLO; GARRIDO; HERNÁNDEZ, 2016; BARBOSA; DA SILVA; 2017; KUMAR; KUMAR, 2018). Sin embargo, hay que tener en cuenta que dicha actitud positiva por parte del profesorado hacia la utilización de las TIC debe venir acompañada de una respectiva formación que contribuya al fortalecimiento de la competencia digital docente (AZNAR; CÁCERES; TRUJILLO; ROMERO, 2019).

En una somera aproximación al concepto, cabe destacar que el germen de la mencionada competencia radica en la Ponencia de la Competencia Digital Docente organizada por el Instituto Nacional de Tecnologías Educativas y de Formación del Profesorado (INTEF, 2017). Su objetivo prioritario fue el de presentar el Marco Común De Competencia Digital Docente (BLANCO, 2018) según los principios establecidos por el Marco estratégico de Educación y Formación y a las recomendaciones estipuladas por el Parlamento Europeo (Comisión Europea, 2016; Comisión Europea, 2009).

Gracias a las acciones llevadas a cabo en la citada ponencia, se consigue estandarizar la competencia digital del profesorado para que pueda ser abordada por toda la comunidad educativa de forma generalizada y bajo un criterio común. Esta estandarización se realiza en seis niveles distintos que aglutinan un total de veintiuna competencias y cinco áreas competenciales organizadas de la siguiente forma: a) Información y Alfabetización informacional; b) Comunicación y Colaboración; c) Creación de Contenido Digital; d) Seguridad en entornos digitales; e) Resolución de problemas (DURÁN; GUTIÉRREZ; PRENDES, 2016; ESTEVE, 2016).

Existe un importante volumen de investigaciones que han constatado un bajo nivel generalizado por parte del profesorado en lo que a competencia digital se refiere (AFANADOR, 2017; FALCÓ, 2017; FERNÁNDEZ; FERNÁNDEZ, 2016; GONZÁLEZ; GUTIÉRREZ, 2017; PÉREZ; RODRÍGUEZ, 2016).

A su vez, también se encuentran investigaciones en las que se concretan que el contacto con la metodología TIC, como es el caso entre ellas de blended learning (ČERNÁ; BORKOVCOVÁ, 2018; PANYAJAMORN et al., 2018), conlleva una mejora en destrezas concretas de la competencia digital, como es el caso de la resolución de problemas o la seguridad digital (REVILLA; ALPISTE; FERNÁNDEZ; SANTOS, 2017; ALENEZI, 2017). El haber contactado con la metodología no mejora solamente la destreza digital del docente, sino que le incentiva a seguir utilizándolas, provocando una mejora en su motivación (LASKARIS; KALOGIANNAKIS; HERETAKIS, 2017).

Sin embargo, resulta especialmente relevante desarrollar un corpus científico que 
indague de forma concreta en el estado de la cuestión con un mayor nivel de concreción para poder conocer las causas concretas del bajo nivel en cada una de las áreas de la competencia digital y así poder encauzar los planes de formación continua hacia acciones eficientes que contemplen la realidad formativa del docente actual.

Además de la idoneidad de los planes de formación continua, es necesario buscar el origen del bajo nivel competencial de los docentes dentro del ámbito digital en la escasez de recursos de un importante número de centros educativos que cuentan con un material desfasado y escaso (AREA; GUARRO, 2012; GALLEGO; GÁMIZ; GUTIÉRREZ, 2010; SUÁREZ; DÍAZ; FERNÁNDEZ, 2012). Por otra parte, resulta especialmente necesario evaluar los planes de formación inicial del alumnado universitario que, en un futuro, pasará a engrosar la nómina de docentes del mercado laboral (RODRÍGUEZGARCÍA; ROMERO; FUENTES, 2019; MARTÍNEZ, 2018; MORENO, GABARDA; RODRÍGUEZ, 2018; PEIRATS; MARÍN; GRANADOS; MOROTE, 2018).

En definitiva, la tecnología ha influido enormemente en el avance del panorama educativo actual, y todos los centros educativos han podido vivenciar de una y otra forma la inclusión de lo tecnológico en el debate educativo. Dentro de los distintos tipos de centros educativos existentes, resulta de especial relevancia destacar la perspectiva de las cooperativas de enseñanza. Estos tipos de centros comparten el escenario educativo con los de naturaleza pública y privada (FERNÁNDEZ; MUÑIZ, 2012), y están viviendo durante los últimos años un especial periodo de crecimiento y evolución (CAMPOS; NAVARRO, 2017).

Las cooperativas de enseñanza establecen entre sus fines prioritarios el ofrecer a su alumnado una enseñanza eficaz y de calidad (LÓPEZ; MORENO; POZO, 2018), centrando sus esfuerzos en la capacitación continua de sus docentes para que puedan poner en práctica metodologías pedagógicas innovadoras (LÓPEZ; FUENTES, 2018). De esta forma, esta tipología de centro educativo prestará especial atención -entre otros aspectos- a la competencia digital de los profesores que integran el colectivo docente para poder alcanzar la mencionada calidad en su servicio.

\section{Material y métodos}

\subsection{Problemática y justificación del estudio}

La incidencia tecnológica en el ámbito escolar ha propiciado la aparición de nuevas corrientes y paradigmas educacionales focalizados en la construcción activa del conocimiento por parte de los discentes, facilitada por los recursos digitales y bajo el seguimiento del profesorado, produciéndose un cambio de roles en los procesos de enseñanza y aprendizaje.

Esta situación implica, por un lado, la dotación de equipamientos innovadores en los centros educativos para hacer frente a las nuevas demandas de la sociedad de la información y el conocimiento y, por otro lado, la formación de los docentes para un eficaz desempeño pedagógico en los nuevos entornos mediados por la tecnología.

Por tanto, las cooperativas de enseñanza -dada su naturaleza innovadora y su preocupación por ofrecer un servicio de calidad- deben estar a la vanguardia de las 
nuevas metodologías emergentes de aprendizaje promovidas por el acercamiento e inclusión progresiva de las TIC en el ecosistema educativo.

La requerida competencia digital de los docentes para asumir el reto de formar a las actuales generaciones de estudiantes y el aprovechamiento de las potencialidades que brindan los recursos y herramientas digitales han sido las razones que han propiciado el despliegue de esta investigación, a raíz del desarrollo del proyecto INNOVACOOP -un proyecto de formación del profesorado en competencia digital, liderado por distintos profesionales del sector de la enseñanza en la Ciudad Autónoma de Ceuta (España)persiguiendo la adecuación y transformación de los centros educativos a las tendencias actuales del panorama escolar.

\subsection{Objetivos}

Como objetivo general, este estudio dirige su atención hacia conocer la mejora de la competencia digital de los docentes de una cooperativa de enseñanza de la Ciudad Autónoma de Ceuta (España), tras la implantación del proyecto INNOVACOOP.

Los objetivos específicos que guían esta investigación son los siguientes:

- Conocer la mejora alcanzada en el área de la información y alfabetización informacional del docente.

- Determinar el avance producido en el área de la comunicación y colaboración de los docentes en medios digitales.

- Averiguar el progreso del profesorado en el área de la creación de contenidos digitales

- Concretar la evolución del área relacionada con la seguridad de los docentes en los entornos digitales.

- Determinar el cambio acontecido en el área de la resolución de problemas tecnológicos.

- Comprobar el estado de la motivación docente antes y después de la implantación del proyecto.

- Conocer las metodologías empleadas por el profesorado en ambos momentos del estudio.

\subsection{Diseño de investigación}

Para el desarrollo del estudio se ha seguido un método de investigación cuantitativo basado en un estudio de caso realizado en una cooperativa de enseñanza. Se ha establecido un diseño experimental de tipo pretest-postest en un solo grupo (RODRÍGUEZ, 2011).

Como variable independiente $(\mathrm{VI})$ se ha establecido un plan de formación del profesorado y como variable dependiente (VD) se ha tomado el nivel de competencia digital de los docentes.

Durante la investigación se efectúa una primera observación $\left(\mathrm{O}_{1}\right)$, seguidamente se 
aplica un tratamiento $(X)$ y se concluye con una segunda y última medición $\left(\mathrm{O}_{2}\right)$ con la finalidad de conocer el alcance de la intervención llevada a cabo. Por tanto, el estudio se concreta en el siguiente esquema:

$\mathrm{O}_{1} \rightarrow$ Aplicación de la prueba de medición competencial (Pretest).

$\mathrm{X} \rightarrow$ Suministro del tratamiento/intervención (VI).

$\mathrm{O}_{2} \rightarrow$ Aplicación de la prueba de medición competencial (Postest).

El análisis de datos se ha efectuado a nivel descriptivo y correlacional, por medio del programa Statistical Package for the Social Sciences (SPSS) v.22, marcando un $p<0.05$ como diferencia estadísticamente significativa en el estudio de las variables. Se han empleado estadísticos como la media $(M e)$ y la desviación típica $(D t)$. Para la comparación de medias entre pretest-postest se ha empleado la prueba $t$-Student, estableciendo el valor de independencia en base al nivel de significancia del $p$-valor. Asimismo, se ha aplicado el estadístico $d$ de Cohen y la correlación biserial $(r$ ) para determinar el tamaño del efecto (LANDERO; GONZÁLEZ, 2006).

\subsection{Muestra}

Los sujetos que participan en esta investigación son docentes $(n=30)$ de una cooperativa de enseñanza de la Ciudad Autónoma de Ceuta (España). Para la confección de la muestra se realizó un muestreo aleatorio simple (VIVANCO; 2005) en toda la población ( $\mathrm{N}=45)$ del centro educativo en cuestión.

Las principales características de los docentes participantes en este estudio se encuentran recogidas en las Figuras 1 y 2, de elaboración propia, al igual que la totalidad de tablas y figuras del presente trabajo.

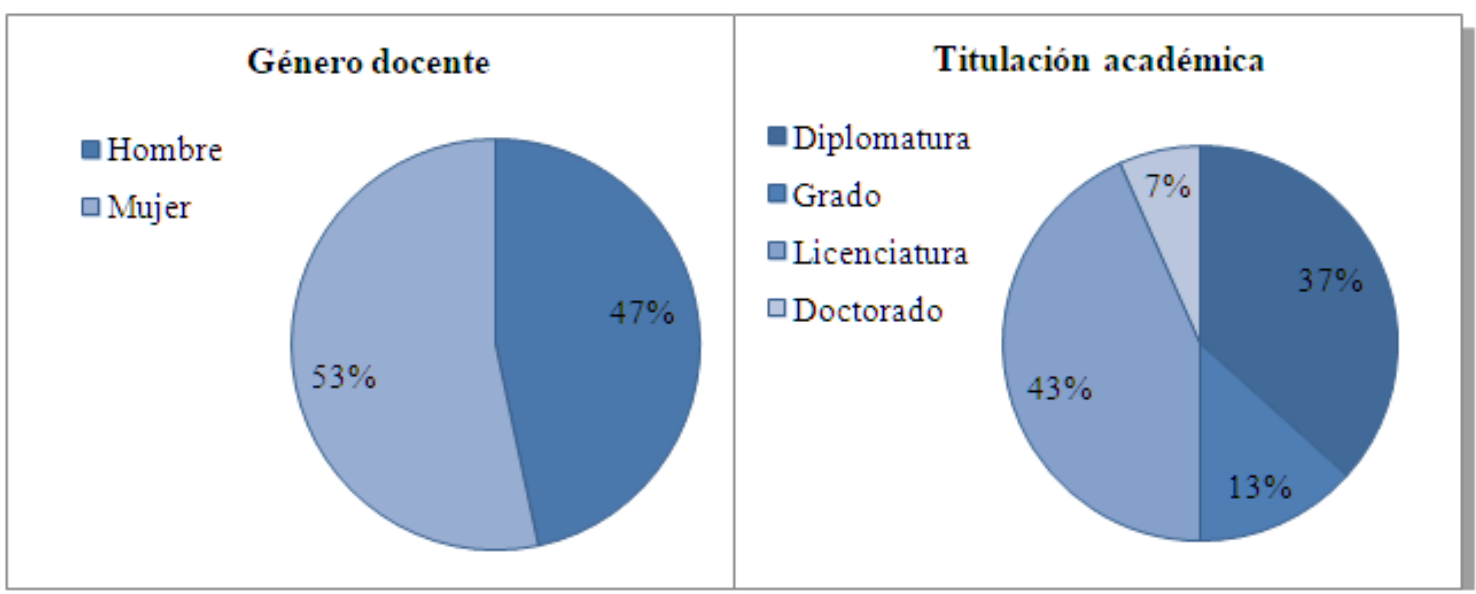

Figura 1: Género docente y titulación académica de la muestra. 

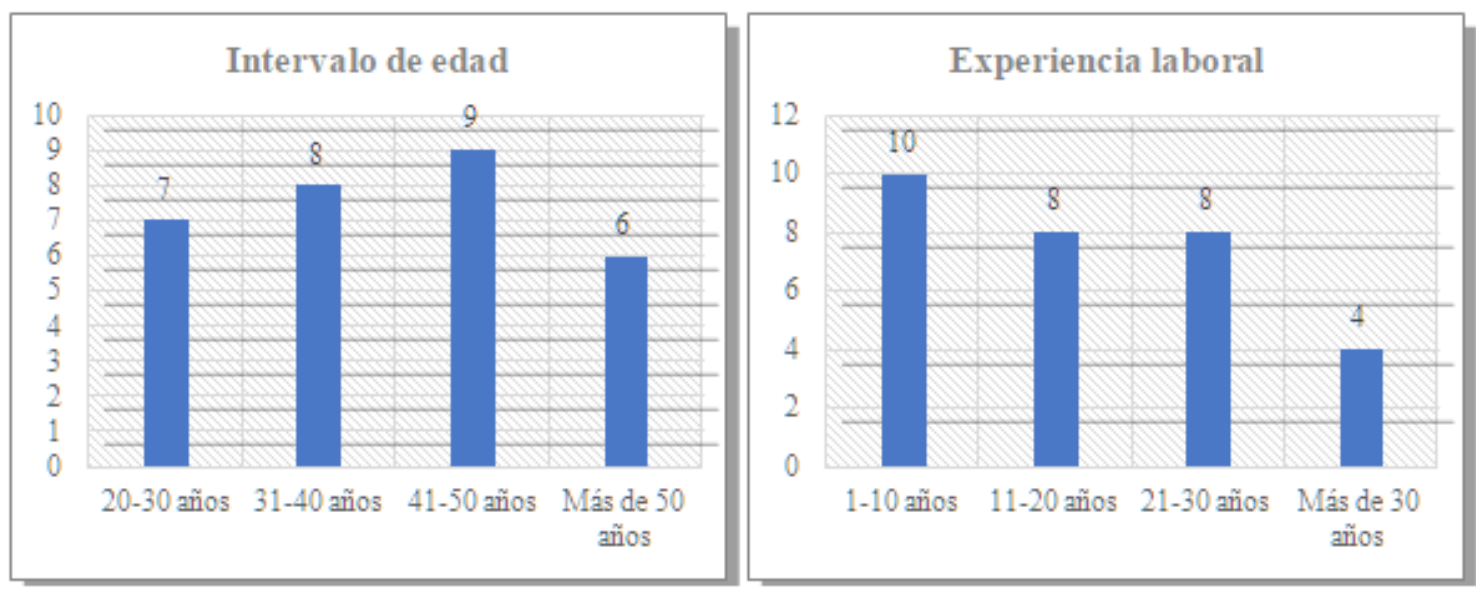

Figura 2: Edad y experiencia laboral de la muestra.

\subsection{Instrumento}

Se ha recurrido al método por encuesta para desarrollar el proceso de recogida de datos (QUINTANAL; RIESCO; FERNÁNDEZ; SÁNCHEZ, 2012). Para ello se ha confeccionado un cuestionario ad hoc, articulado en diferentes dimensiones con sus respectivas variables que dan forma al instrumento, con la intención de abarcar todos los requerimientos de la investigación (ALAMINOS; CASTEJÓN, 2006).

El cuestionario integra un total de 75 ítems, encuadrados en 7 dimensiones y 30 variables de estudio. El formato de respuesta se ha configurado -en su mayoría- en escala Likert (1-nada, 2-poco, 3-bastante y 4-totalmente) y otras de respuesta cerrada.

Los índices de fiabilidad y validez del instrumento se hallaron por un juicio de expertos compuesto por 4 doctores especialistas en la temática y pertenecientes a las Universidades de Granada, Sevilla y Málaga (España), quienes consideraron la validez de contenido del cuestionario. Acto seguido de la validación cualitativa, se inició un análisis multivariante para simplificar los datos sin necesidad de perder información, por medio del Análisis de Componentes Principales (ACP), llevado a cabo a través del test de KaiserMeyer-Olkin (KMO), cuyo resultado fue de $p=0.913$ y el test de esfericidad de Bartlett, con un $p=0.0005$. Ambas pruebas reflejaron un ACP pertinente. De manera complementaria y para mejorar la interpretación de los datos, se efectuó una rotación oblicua Promax con normalización Kaiser (PÉREZ, 2005).

La fiabilidad fue obtenida mediante el estadístico alfa $(\alpha)$ de Cronbach, cuyo resultado de la prueba arrojó un valor de $\alpha=0.876$, etiquetando al instrumento como altamente fiable (GEORGE; MALLERY, 2003).

Tras analizar el cuestionario, se realizó una prueba piloto como recomienda Corral (2009), en un grupo de sujetos diferentes a la muestra de estudio, con el propósito de optimizar el instrumento y reducir la aparición de sesgos y problemas de comprensión que provoquen una alteración de los resultados que se obtengan.

En la Tabla 1 se encuentran las principales características del instrumento utilizado. 
Tabla 1: Peculiaridades del cuestionario.

\begin{tabular}{|c|c|c|c|}
\hline Dimensión & Variables & Ítems & Alfa de Cronbach \\
\hline $\begin{array}{l}\text { Sociodemográfica } \\
\text { (SOCI) }\end{array}$ & $\begin{array}{c}\text { Género (GEN) } \\
\text { Edad (ED) } \\
\text { Titulación académica (TITUL) } \\
\text { Experiencia docente (EXPE) }\end{array}$ & 4 & $\alpha=0.857$ \\
\hline $\begin{array}{l}\text { Información y } \\
\text { alfabetización } \\
\text { informacional } \\
\text { (INAIN) }\end{array}$ & $\begin{array}{c}\text { Identificación (IDEN) } \\
\text { Localización (LOCA) } \\
\text { Recuperación (RECU) } \\
\text { Almacenamiento (ALMA) } \\
\text { Organización (ORGA) } \\
\text { Análisis (ANA) } \\
\text { Evaluación (EVAL) }\end{array}$ & 18 & $\alpha=0.894$ \\
\hline $\begin{array}{l}\text { Comunicación y } \\
\text { colaboración } \\
\text { (COMCOL) }\end{array}$ & $\begin{array}{l}\text { Comunicación (COMU) } \\
\text { Conexión (CONEX) } \\
\text { Colaboración (COL) } \\
\text { Interacción (INTER) } \\
\text { Participación (PARTI) }\end{array}$ & 12 & $\alpha=0.869$ \\
\hline $\begin{array}{l}\text { Creación de } \\
\text { contenidos digitales } \\
\text { (CRECON) }\end{array}$ & $\begin{array}{c}\text { Creación (CREA) } \\
\text { Edición (EDI) } \\
\text { Integración (INTE) } \\
\text { Programación (PROG) } \\
\text { Derechos de uso (DER) }\end{array}$ & 14 & $\alpha=0.882$ \\
\hline Seguridad (SEG) & $\begin{array}{l}\text { Protección personal (PROPER) } \\
\text { Protección de datos (PRODA) } \\
\text { Protección identidad digital (PROIDI) } \\
\text { Uso seguro y crítico (SECRI) }\end{array}$ & 15 & $\alpha=0.874$ \\
\hline $\begin{array}{l}\text { Resolución de } \\
\text { problemas } \\
\text { (RESOL) }\end{array}$ & $\begin{array}{l}\text { Reconocimiento del problema } \\
\text { (RECO) } \\
\text { Toma de decisiones (DECI) } \\
\text { Resolución técnica (RESOLT) }\end{array}$ & 7 & $\alpha=.862$ \\
\hline $\begin{array}{c}\text { Desempeño } \\
\text { docente (DESDO) }\end{array}$ & $\begin{array}{l}\text { Metodología (METOD) } \\
\text { Motivación (MOTIV) }\end{array}$ & 5 & $\alpha=0.896$ \\
\hline
\end{tabular}

\subsection{Procedimiento}

La investigación se inició en el mes de octubre de 2017 y se estructuró en cuatro fases. En primer lugar, se tomó contacto con el equipo directivo del centro educativo para reflejar el interés de los investigadores en llevar a cabo un estudio en una cooperativa de enseñanza con la intención de medir la mejora de la competencia digital de los docentes tras la puesta en marcha de un programa formativo de ámbito tecnológico e innovador.

Tras la aceptación y la obtención de los permisos necesarios, se efectuó la primera medición para conocer el punto de partida, referido al nivel de conocimientos, destrezas y aptitudes del profesorado en su vertiente digital.

A continuación, una vez recabados los datos, se produjo el desarrollo de un plan de formación bajo una modalidad blended learning o mixta, combinando una enseñanza presencial con una virtual (BOELENS; DE WEVER; VOET, 2017). Todo ello a través de un programa de formación del profesorado en ambientes digitales perteneciente a la 
Dirección provincial del Ministerio de Educación y Formación Profesional, en el que se impartieron contenidos - de manera presencial - relacionados con la competencia digital y su aplicación pedagógica, distribuidos en 8 sesiones, haciendo un total de 24 horas lectivas, además del seguimiento de los contenidos y otras actividades en entornos digitales por medio de la plataforma Moodle, con una estimación de 10 horas.

Por último, se llevó a cabo la segunda medición con la finalidad de comprobar si tal ejercicio de formación desplegado produjo mejoras en la competencia digital del profesorado. Todos los datos obtenidos sirvieron para realizar un análisis estadístico en profundidad, permitiendo ofrecer unos resultados que dieran respuesta a los objetivos planteados.

\section{Resultados}

A continuación, se presentan los principales resultados obtenidos en la realización de este estudio. Comenzando por el análisis de la dimensión vinculada con la información y alfabetización informacional de los docentes (Tabla 2), los procedimientos estadísticos han puesto de manifiesto que los profesionales de la educación que han participado en este estudio han sufrido una mejora en sus habilidades y destrezas en esta área de la competencia digital tras la aplicación de un plan formativo. Concretamente las variables EVAL e IDEN son las que han alcanzado un mayor nivel de progreso con respecto a su evaluación inicial. Del mismo modo, tras la realización de la prueba $t$-Student y la obtención del $p$-valor, se revela que a excepción de la variable ORGA, el resto ha resultado estadísticamente significativo dados los valores de $p<0.05$. En cuanto al tamaño del efecto logrado en las variables, es EVAL la que ha adquirido un mayor efecto en la misma según las cifras mostradas en la prueba $d$ de Cohen y la correlación biserial.

Tabla 2: Resultados obtenidos para la dimensión INAIN.

\begin{tabular}{|c|c|c|c|c|c|c|c|}
\hline & \multicolumn{2}{|c|}{ Grupo, Me (Dt) } & \multirow{2}{*}{$M e_{1}-M e_{2}$} & \multicolumn{2}{|c|}{$\begin{array}{l}\text { Prueba } t- \\
\text { Student }\end{array}$} & \multirow{2}{*}{$-d$} & \multirow{2}{*}{$-r$} \\
\hline & $\begin{array}{r}\text { Pretest }(\mathrm{n}= \\
30)\end{array}$ & $\begin{array}{r}\text { Postest }(\mathrm{n}= \\
30)\end{array}$ & & $t(87)$ & $p$-valor & & \\
\hline IDEN & $2.08(1.038)$ & $2.76(1.091)$ & -0.68 & -2.47 & 0.016 & 0.638 & 0.304 \\
\hline LOCA & $2.28(0.981)$ & $2.92(0.997)$ & -0.64 & -2.51 & 0.015 & 0.647 & 0.307 \\
\hline RECU & $1.92(0.997)$ & $2.56(1.003)$ & -0.64 & -2.48 & 0.016 & 0.639 & 0.305 \\
\hline ALMA & $2.84(0.987)$ & $3.28(0.737)$ & -0.44 & -1.96 & 0.055 & 0.505 & 0.245 \\
\hline ORGA & $1.96(1.06)$ & $2.36(0.995)$ & -0.4 & -1.51 & 0.137 & 0.389 & 0.191 \\
\hline ANA & $2.24(1.091)$ & $2.84(0.85)$ & -0.6 & -2.38 & 0.021 & 0.613 & 0.293 \\
\hline EVAL & $2.28(1.061)$ & $3.04(0.841)$ & -0.76 & -3.07 & 0.003 & 0.793 & 0.369 \\
\hline
\end{tabular}

En el estudio de la dimensión concerniente a la comunicación y colaboración entre los docentes en medios digitales, la Tabla 3 muestra las valoraciones alcanzadas - en esta segunda área de la competencia digital docente - antes y después del programa de intervención efectuado al respecto. Como se aprecia, la variable COMU ha revelado un mayor progreso con respecto a las demás variables de esta dimensión. De igual forma, la 
$t$-Student y el $p$-valor realizado otorgan significancia estadística en las variables COMU, CONEX y PARTI $(p<0.05)$. Sobre el tamaño del efecto conseguido en las distintas variables estudiadas en la dimensión COMCOL, la variable COMU es la que ha obtenido un mayor tamaño tras las pruebas pertinentes ( $d$ de Cohen; $r$ ).

En cuanto al análisis de la dimensión conexa a la creación de contenidos digitales por los docentes (segunda mitad de la Tabla 3), la realización del plan de formación como tratamiento de intervención en esta investigación ha originado mejoras en las distintas variables de la citada dimensión, siendo CREA y EDI las variables que han conseguido mayor proyección. Los procedimientos estadísticos ( $t$-Student y $p$-valor) llevados a cabo han establecido diferencias estadísticamente significativas en todas las variables de la dimensión CRECON, excepto en la variable DER. En alusión a las pruebas $d$ de Cohen y la correlación biserial, la variable que mayor tamaño del efecto ha obtenido es EDI.

Tabla 3: Resultados obtenidos para las dimensiones COMCOL y CRECON.

\begin{tabular}{|c|c|c|c|c|c|c|c|}
\hline & \multicolumn{2}{|c|}{ Grupo, Me (Dt) } & \multirow[b]{2}{*}{$M e_{1}-M e_{2}$} & \multicolumn{2}{|c|}{ Prueba $t$-Student } & \multirow[b]{2}{*}{$-d$} & \multirow[b]{2}{*}{$-r$} \\
\hline & $\begin{array}{l}\text { Pretest } \\
(\mathrm{n}=30)\end{array}$ & $\begin{array}{r}\text { Postest (n } \\
=30)\end{array}$ & & $t(87)$ & $p$-valor & & \\
\hline COMU & $\begin{array}{r}2.08 \\
(0.997)\end{array}$ & $3.12(0.881)$ & -1.04 & -4.28 & $<0.001$ & 1.105 & 0.483 \\
\hline CONEX & $\begin{array}{r}2.24 \\
(0.97)\end{array}$ & $2.96(1.02)$ & -0.72 & -2.8 & 0.007 & 0.723 & 0.341 \\
\hline COL & $\begin{array}{r}2.56 \\
(1.003)\end{array}$ & $2.84(0.943)$ & -0.28 & -1.11 & 0.271 & 0.402 & 0.197 \\
\hline INTER & $\begin{array}{r}2.44 \\
(1.003)\end{array}$ & $2.8(1.08)$ & -0.36 & -1.34 & 0.186 & 0.345 & 0.171 \\
\hline PARTI & $\begin{array}{r}2.32 \\
(0.901)\end{array}$ & $3.08(0.812)$ & -0.76 & -3.43 & 0.001 & 0.886 & 0.405 \\
\hline CREA & $\begin{array}{r}2.24 \\
(1.052)\end{array}$ & $2.96(0.841)$ & -0.72 & -2.93 & 0.005 & 0.756 & 0.353 \\
\hline EDI & $\begin{array}{r}2.28 \\
(1.021)\end{array}$ & $3.01(0.816)$ & -0.72 & -3.02 & 0.004 & 0.789 & 0.367 \\
\hline INTE & $\begin{array}{r}1.92 \\
(0.954)\end{array}$ & $2.44(0.917)$ & -0.52 & -2.15 & 0.036 & 0.555 & 0.267 \\
\hline PROG & $\begin{array}{r}1.48 \\
(0.823)\end{array}$ & $1.96(0.935)$ & -0.48 & -2.11 & 0.039 & 0.544 & 0.263 \\
\hline DER & $\begin{array}{r}2.32 \\
(0.988)\end{array}$ & $2.6(0.913)$ & -0.28 & -1.14 & 0.259 & 0.294 & 0.145 \\
\hline
\end{tabular}

Referente a la dimensión relacionada con el área de la seguridad de los docentes en el plano digital (Tabla 4), se obtiene que todas las variables han experimentado una mejoría, pero son las variables PRODA y PROPER las que han alcanzado un mayor progreso tras el desarrollo del programa formativo. Además, todas las variables de la citada dimensión han reflejado significancia estadística, verificada en los valores obtenidos en el test $t$-Student y $p$-valor, al hallarse en este último cifras de $p<0.05$. En alusión a la fuerza del fenómeno producido, las pruebas $d$ de Cohen y $r$ han revelado un tamaño del efecto pertinente, sobre todo en la variable PRODA.

Respecto a la dimensión concomitante con el área de la resolución de problemas por parte de los docentes, la Tabla 4 muestra leves progresos en las distintas variables que componen esta dimensión, reflejado en la diferencia producida entre la primera y 
segunda medición $\left(M_{1}-M e_{2}\right)$, siendo la variable DECI seguida - muy de cerca - por RESOLT las mínimamente destacadas. Prueba de ello es que no se han alcanzado diferencias estadísticamente significativas en los procedimientos estadísticos efectuados. Asimismo, el tamaño del efecto producido, visto en los valores de $d$ y $r$, es considerado pequeño.

Sobre la variable MOTIV perteneciente a la dimensión DESDO, como se aprecia al final de la Tabla 4, tras la aplicación del plan formativo la motivación docente ha experimentado una mejora considerable, revelando las pruebas realizadas significancia estadística y una fuerza del fenómeno pertinente.

Tabla 4: Resultados de las dimensiones SEG y RESOL y de la variable MOTIV.

\begin{tabular}{|c|c|c|c|c|c|c|c|}
\hline & \multicolumn{2}{|c|}{ Grupo, Me (Dt) } & \multirow[b]{2}{*}{$M e_{1}-M e_{2}$} & \multicolumn{2}{|c|}{ Prueba $t$-Student } & \multirow[b]{2}{*}{$-d$} & \multirow[b]{2}{*}{$-r$} \\
\hline & $\begin{array}{c}\text { Pretest } \\
(n=30)\end{array}$ & $\begin{array}{l}\text { Postest } \\
(n=30)\end{array}$ & & $t(87)$ & $p$-valor & & \\
\hline PROPER & $\begin{array}{r}1.84 \\
(0.943)\end{array}$ & $\begin{array}{r}2.56 \\
(0.821)\end{array}$ & -0.72 & -3.15 & 0.002 & 0.814 & 0.377 \\
\hline PRODA & $\begin{array}{r}1.72 \\
(0.843)\end{array}$ & $2.64(0.81)$ & -0.92 & -4.31 & $<0.001$ & 1.113 & 0.486 \\
\hline PROIDI & $\begin{array}{r}1.92 \\
(0.997)\end{array}$ & $\begin{array}{r}2.52 \\
(0.963)\end{array}$ & -0.6 & .37 & 0.021 & 0.612 & 0.292 \\
\hline SECRI & $\begin{array}{r}2.28 \\
(0.843) \\
\end{array}$ & $2.8(0.866)$ & -0.52 & -2.36 & 0.022 & 0.608 & 0.291 \\
\hline RECO & $\begin{array}{r}\overline{2} . \overline{44} \\
(0.917)\end{array}$ & $2.6(0.913)$ & -0.16 & -0.68 & 0.501 & 0.175 & 0.087 \\
\hline DECI & $\begin{array}{r}2.24 \\
(0.926)\end{array}$ & $\begin{array}{r}2.52 \\
(0.872)\end{array}$ & -0.28 & -1.21 & 0.233 & 0.311 & 0.153 \\
\hline RESOLT & $\begin{array}{r}2.28 \\
(0.891)\end{array}$ & $\begin{array}{r}2.48 \\
(0.872)\end{array}$ & -0.2 & -0.88 & 0.383 & 0.226 & 0.112 \\
\hline MOTIV & $\begin{array}{r}2.36 \\
(1.036)\end{array}$ & $\begin{array}{r}3.01 \\
(1.001)\end{array}$ & -0.64 & 2.43 & 0.018 & 0.638 & 0.304 \\
\hline
\end{tabular}

La Figura 3 muestra el tipo de metodología utilizada por los docentes antes y después de la puesta en marcha del plan para la formación docente en competencia digital. Se aprecia que las metodologías con más inclinación hacia un uso potencial de las TIC revelan mejores resultados en la segunda medición realizada que coincide con la finalización del proyecto de formación docente, siendo la metodología combinada con un uso preferente a las TIC la que ha obtenido un mayor contraste. 


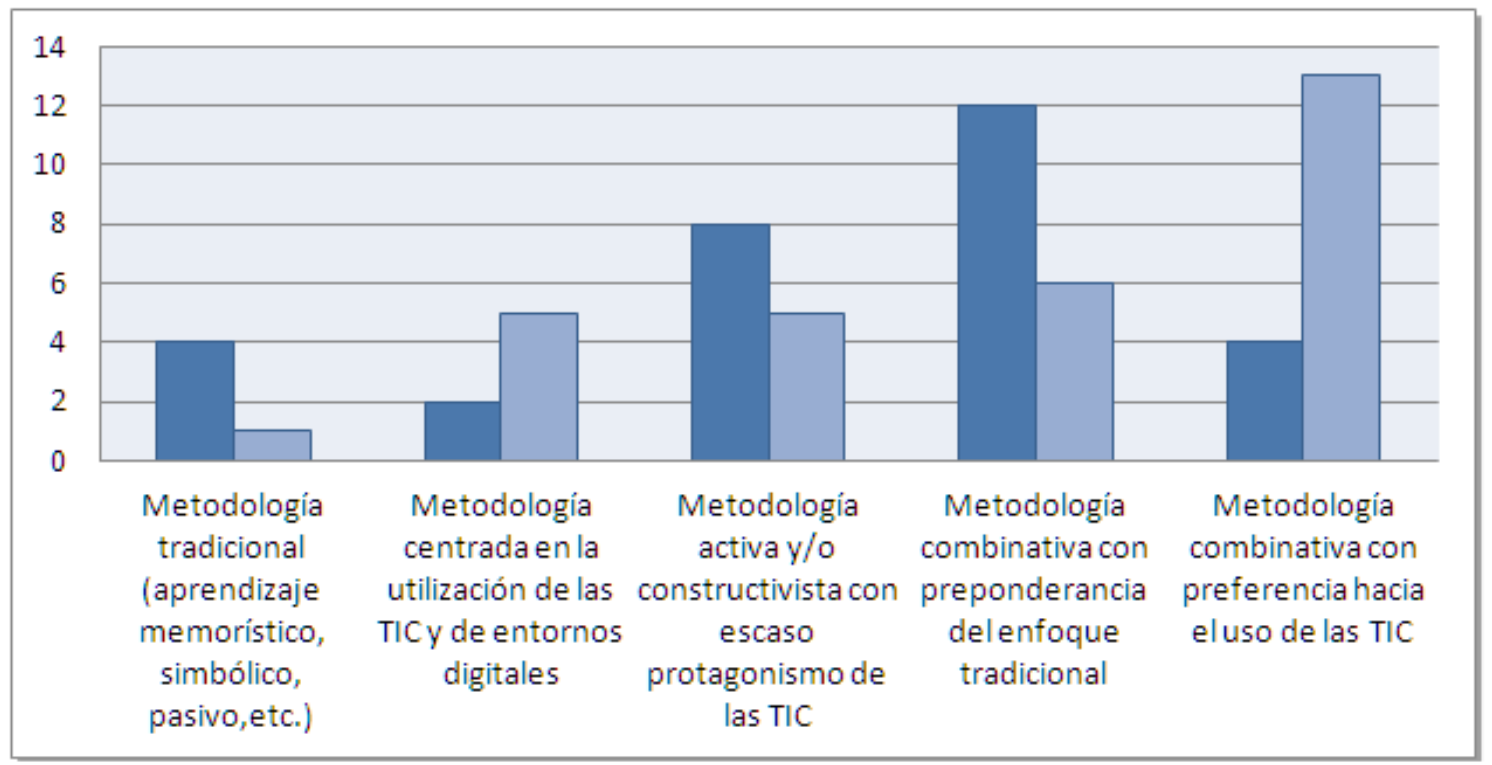

Figura 3: Metodología docente empleada antes y después de la VI.

\section{Discusión y conclusiones}

El desarrollo de la competencia digital sigue siendo un objetivo que la comunidad docente debe seguir abordando. Los resultados del presente estudio reflejan que la aplicación de proyectos autonómicos que apoyen y contribuyan en infraestructura, recursos y formación digital tiene una incidencia positiva en el cuerpo del profesorado en las respectivas dimensiones que conforman la competencia digital.

Se confirma la premisa presentada en investigaciones anteriores (ÁREA: GUARRO, 2012; GALLEGO; GÁMIZ; GUTIÉRREZ, 2010; SUÁREZ; ALMERICH; DÍAZ; FERNÁNDEZ, 2012) en la que se constata la necesidad de que los equipos gubernamentales subvencionen a los centros con recursos digitales y tecnológicos -a través de proyectos de innovación- con la finalidad de fomentar su utilización.

Esta idea queda reflejada en los resultados del presente estudio, en el cual las medidas postest una vez aplicada la metodología blended learning muestran una mejora generalizada en cada una de las destrezas digitales, siendo coincidente con otros resultados similares que aplicaron este método en contextos educativos (ČERNÁ; BORKOVCOVÁ, 2018; PANYAJAMORN et al., 2018).

Si procedemos a concretar su desarrollo, resulta preciso destacar el progreso de los docentes en áreas como la seguridad, un ámbito que es especialmente importante, debido al temor que provoca el navegar por el mundo digital y la cantidad de riesgos que existen en la plataforma red. Sin duda, el avance significativo en esta dimensión supondrá un punto de partida para incentivar las TIC en la enseñanza del cuerpo docente (ALENEZI, 2017).

A su vez, se observó que la implementación del plan de formación del profesorado planteada por el proyecto INNOVACOOP tuvo como resultado un progreso óptimo en los docentes en alfabetización informacional, así como en comunicación y colaboración y en la creación de contenidos digitales. De esta manera, se constata la necesidad de seguir 
abordando la formación digital continua en el profesorado de las diferentes etapas educativas, puesto que cuanto más se incida en una formación de calidad que enseñe las numerosas vías de aprendizaje que tienen las TIC, mayor utilización por parte del cuerpo docente se le otorgará (AFANADOR, 2017; FALCÓ, 2017; FERNÁNDEZ; FERNÁNDEZ, 2016).

Referente a la resolución de problemas, el presente estudio indica que el cuerpo docente tuvo una mejora leve, que por tanto, debe seguir siendo un terreno aún por investigar en su mejora. Se coincide con Revilla Muñoz et al. (2017) en la afirmación sobre la dificultad existente en el profesorado respecto a la resolución de problemas en la competencia digital.

Por último, se constata que el contacto con la metodología blended learning provocó no solamente una mejora competencial en el profesorado, sino que incrementó su motivación hacia la tecnología y sus implicaciones educativas, resultado coincidente con el estudio de Laskaris et al (2017).

En suma, atendiendo a los datos recogidos, podemos dilucidar varias conclusiones:

- El trabajo sobre el desarrollo de la competencia digital aún es extenso, pese a las numerosas investigaciones que comprenden su evaluación y desarrollo, pues se trata de un trabajo ambicioso y en el que numerosos factores entran en juego para su óptimo desempeño.

- Entre estos factores, se encuentra el apoyo autonómico y estatal, que debe contemplarse y hacerse presente a través de proyectos como INNOVACOOP, a través del cual se proporcionen recursos digitales al centro, así como una formación de calidad al profesorado.

- Esta formación debe estar dirigida en la utilización de metodologías en la que la tecnología sea protagonista, tal y como ha sido el caso de blended learning. Tal y como se ha visualizado en el presente estudio, el contacto con este método didáctico ha conllevado una mejora del cuerpo docente analizado en las distintas dimensiones que componen la competencia digital, así como en su motivación hacia el uso de las TIC y metodologías que las implican en el aula.

- Por lo tanto, se debe de incidir en plantear investigaciones que fomenten el contacto directo con la tecnología y su modo de aplicarlo en el aula, con el objeto de promover un cambio actitudinal en el profesorado hacia las TIC, para lograr un mayor uso de las mismas en el aula.

La principal limitación que se ha producido en el desarrollo de la investigación se focaliza en la escasa colaboración de determinados participantes durante el proceso de recogida de datos a través del cuestionario y en ambos momentos de medición llevados a cabo para desarrollar el diseño pre-post, pues ciertos sujetos no cumplieron el plazo establecido para cumplimentar todos y cada uno de los ítems del instrumento, lo que desembocó en un retraso en los procedimientos investigativos.

Como futura línea de estudio se pretende realizar un estudio comparativo entre docentes de distintos tipos de centros educativos, realizando una triangulación entre centros públicos, privados y concertados, con el propósito de concretar si el tipo de centro 
es un factor determinante en el desarrollo de la competencia digital de los docentes.

\section{Referências}

AFANADOR, H. A. Estado actual de las competencias TIC de docentes. Puente, 9(2), p. 23-32, 2017.

ALAMINOS, A.; CASTEJÓN, J. L. Elaboración, análisis e interpretación de encuestas, cuestionarios y escalas de opinión. Alicante: Marfil, 2006.

ALENEZI, A. Obstacles for teachers to integrate technology with instruction. Education and Information Technologies, 22(4), p. 1797-1816, 2017.

AREA, M. La alfabetización digital y la formación de la ciudadanía del siglo XXI. Revista Integra Educativa, 7(3), p. 21-33, 2015.

AREA, M.; GUARRO, A. La alfabetización informacional y digital: fundamentos pedagógicos para la enseñanza y el aprendizaje competente. Revista española de documentación científica, 35, p. 46-74, 2012.

ARZOLA, D.; LOYA, C.; GONZÁLEZ, A. El trabajo directivo en educación primaria: liderazgo, procesos participativos y democracia escolar. IE Revista De InvestigacióN Educativa De La REDIECH, 7(12), p. 35-41, 2017.

AZNAR, I.; CÁCERES, M. P.; TRUJILLO, J. M.; ROMERO, J. M. Impacto de las apps móviles en la actividad física: un meta-análisis. Retos, 36, p. 52-57, 2019.

BARBOSA, T.; NÁVEA DA SILVA, M. Z. Ensinar francês por dispositivos móveis: uma experiência com Duolingo e Whatsapp. Texto livre, v. 10, n. 2, p. 206-219, 2017.

BLANCO, S. M. Marco Común de Competencia Digital Docente. Revista Iberoamericana de Educación a Distancia, 21(1), p. 369-370, 2018.

BOELENS, R.; DE WEVER, B.; VOET, M. Four key challenges to the design of blended learning: A systematic literature review. Educational Research Review, 22, p. 1-18, 2017.

CABERO, J. La formación en la era digital: ambientes enriquecidos por la tecnología. Revista Gestión de la Innovación en Educación Superior, 2(2), p. 41-64, 2017.

CAMPOS, V.; NAVARRO, C. Participación en redes organizacionales y uso de las tecnologías de la información y la comunicación, un estudio de su impacto en los resultados de las cooperativas de enseñanza de la comunidad valenciana. REVESCO. Revista de Estudios Cooperativos, (124), p. 32-46, 2017.

ČERNÁ, M.; BORKOVCOVÁ, A. Blended Learning Concept in Selected Tourism 
Management e-Courses with Focus on Content Development Including Recommender System. Lecture Notes in Computer Science (including subseries Lecture Notes in Artificial Intelligence and Lecture Notes in Bioinformatics), 10949 LNCS, p. 175-187, 2018.

COMISIÓN EUROPEA. Marco estratégico Educación y Formación 2020 (ET2020). Euridyce España. 2009. Recuperado de http://www.mecd.gob.es/educacionmecd/mc/redie-eurydice/prioridades-europeas/et2020.html. Acceso en: 25 jul. 2019.

COMISIÓN EUROPEA. Education and training.Monitor 2016. Portugal. 2016. Recuperado de https://ec.europa.eu/education/sites/education/files/monitor2016-pt_en.pdf. Acceso en: 25 jul. 2019.

CORRAL, Y. Validez y confiabilidad de los instrumentos de investigación para la recolección de datos. Revista Ciencias de la Educación, 19(33), p. 228-247, 2009.

DURÁN, M.; GUTIÉRREZ, I.; PRENDES, M. P. Análisis conceptual de modelos de competencia digital del profesorado universitario. RELATEC: Revista Latinoamericana de Tecnología Educativa, 15(1), p. 97-114, 2016.

ESTEVE, F. Bolonia y las TIC: de la docencia 1.0 al aprendizaje 2.0. La cuestión universitaria, (5), p. 58-67, 2016.

FALCÓ, J. M. Evaluación de la competencia digital docente en la Comunidad Autónoma de Aragón. Revista electrónica de investigación educativa, 19(4), p. 73-83, 2017.

FERNÁNDEZ, F. J.; FERNÁNDEZ, M. J. Generation Z's Teachers and their Digital Skills= Los docentes de la Generación Z y sus competencias digitales. Comunicar, 24(46), p. 97105, 2016.

FERNÁNDEZ, R.; MUÑIZ, M. Colegios concertados y selección de escuela en España: un círculo vicioso. Presupuesto y gasto público, 67, p. 97-118, 2012.

GALLEGO, M. J.; GÁMIZ, V.; GUTIÉRREZ, E. El futuro docente ante las competencias en el uso de las tecnologías de la información y comunicación para enseñar. EDUTEC, Revista de Tecnología Educativa, 34, p. 1-18, 2010.

GEORGE, D.; MALLERY, P. SPSS for Windows step by step: A Simple Guide and Reference. Boston: Allyn \& Bacon, 2003.

GONZÁLEZ, A. Dinamización tecnológica de la escuela a través del liderazgo del coordinador TIC. Estudios pedagógicos, 43(2), p. 115-125, 2017.

GONZÁLEZ, R.; GUTIÉRREZ, A. Competencias mediática y digital del profesorado e integración curricular de la tecnologías digitales. Revista Fuentes, 19(2), p. 57-67, 2017.

INTEF. Marco de Competencia Digital. Madrid: Ministerio de Educación, Ciencia y Deportes, 2017. 
KUMAR, A.; KUMAR, G. The Role of ICT in Higher Education for the 21st Century: ICT as A Change Agent for Education. Multidisciplinary Higher Education, Research, Dynamics \& Concepts: Opportunities \& Challenges For Sustainable Development, 1(1), p. 76-83, 2018.

LANDERO, R.; GONZÁLEZ, M. Estadística con SPSS y metodología de la investigación. México: Trillas, 2006.

LASKARIS, D.; KALOGIANNAKIS, M.; HERETAKIS, E. «Interactive evaluation» of an elearning course within the context of blended education. International Journal of Technology Enhanced Learning, 9(4), p. 339-353, 2017.

LÓPEZ, J.; MORENO, A. J.; POZO, S. Influencia del género y la edad en la formación continua multidisciplinar de los docentes de cooperativas de enseñanza. Revista Innova, 3(8), 42-59, 2018.

LÓPEZ, J.; FUENTES, A. El liderazgo aplicado a los modelos diferenciados en educación: El caso de las cooperativas de enseñanza. En: GAIRÍN, J., \& MERCADER, C. (ed.), Liderazgo y gestión del talento en las organizaciones. Madrid, España: Wolters Kluwer, 2018. p. 169-175.

MACÍAS, E.; CHUM, S.; ARAY, C.; RODRÍGUEZ, C. Liderazgo académico: Estilos y perfiles de gestión en las instituciones de educación superior. ReHuSo: Revista de Ciencias Humanísticas y Sociales, 3(1), p. 82-105, 2018.

MARTÍNEZ, A. Percepciones sobre las competencias de formación básica en Educación Infantil. Aula de Encuentro, 20(1), p. 54-73, 2018.

MCGILL, T.; KOPPI, T.; ARMAREGO, J. ICT industry involvement with ICT education and research in universities: Industry perceptions. Innovation in Teaching and Learning in Information and Computer Sciences, 0(0), 1-18, 2014.

MÉNDEZ J.; DELGADO, M. Las TIC en centros de Educación Primaria y Secundaria de Andalucía. Un estudio de casos a partir de buenas prácticas. Digital Education Review, (29), p. 134-165, 2016.

MORENO, M. D.; GABARDA, V.; RODRÍGUEZ, A. M. Alfabetización informacional y competencia digital en estudiantes de magisterio. Profesorado, Revista de currículum y formación del profesorado, 22(3), p. 253-270. 2018.

MURILLO, F. J.; GARRIDO, C. M.; HERNÁNDEZ, R. Decálogo para una enseñanza eficaz. REICE. Revista Iberoamericana sobre Calidad, Eficacia y Cambio en Educación, 9(1), p. 7-27, 2016.

MURILLO, F. J.; KRICHESKY, G. J. Mejora de la Escuela: Medio siglo de lecciones aprendidas. REICE. Revista Iberoamericana sobre Calidad, Eficacia y Cambio en Educación, 13(1), p. 69-102, 2015. 
PANYAJAMORN, T.; SUANMALI, S.; KOHDA, Y.; CHONGPHAISAL, P.; SUPNITHI, T. Effectiveness of E-Learning Design in Thai Public Schools. Malaysian Journal of Learning and Instruction, 15(1), p. 1-34, 2018.

PEIRATS, J. P.; MARÍN, D. M.; GRANADOS, J. G.; MOROTE, D. M. Competencia digital en los planes de estudios de universidades públicas españolas. REDU: Revista de Docencia Universitaria, 16(1), 4, 2018.

PÉREZ, A.; RODRÍGUEZ, M. J. Evaluación de las competencias digitales autopercibidas del profesorado de Educación Primaria en Castilla y León (España). Revista de Investigación Educativa, 34(2), p. 399-415, 2016.

PÉREZ, C. Métodos estadísticos avanzados con SPSS. Madrid: Thomson, 2005.

POZUELO, J. ¿Y si enseñamos de otra manera? Competencias digitales para el cambio metodológico. Caracciolos. Revista digital de investigación en docencia, 2(1), p. 1-21, 2014.

QUINTANAL, J.; RIESCO, M.; FERNÁNDEZ, E.; SÁNCHEZ, J. C. Fundamentos básicos de metodología de investigación educativa. Madrid: Editorial CCS, 2012.

REVILLA MUÑOZ, O.; ALPISTE PENALBA, F.; FERNÁNDEZ SÁNCHEZ, J.; SANTOS, O. C. Reducing techno-anxiety in high school teachers by improving their ICT problemsolving skills. Behaviour and Information Technology, 36(3), p. 255-268, 2017.

RODRÍGUEZ, E.; PEDRAJA, L. Relación entre el liderazgo transformacional y el clima orientado al servicio de los estudiantes: Evidencia exploratoria desde Chile. Interciencia, 42(10), p. 633-640, 2017.

RODRÍGUEZ, N. Diseños experimentales en educación. Revista de Pedagogía, 32(91), p. 147-158, 2011.

RODRÍGUEZ-GARCÍA, A. M.; ROMERO, J. M.; FUENTES, A. Ampliando fronteras de comunicación y colaboración a través de la red: la competencia digital como medio para promover la interculturalidad académica. Tendencias pedagógicas, 33, p. 59-68, 2019.

SÁNCHEZ, A.; CASTRO, D. Cerrando la brecha entre nativos e inmigrantes digitales a través de las competencias informáticas e informacionales. Apertura, 5(2), p. 6-15, 2013.

STEFANI, G.; ANDRÉS, L.; OANES, E. Transformaciones lúdicas: Un estudio preliminar sobre tipos de juego y espacios lúdicos. Interdisciplinaria, 31(1), p. 39-55, 2014.

SUÁREZ, J. M.; ALMERICH, G.; DÍAZ, I.; FERNÁNDEZ, R. Competencias del profesorado en las TIC. Influencia de factores personales y contextuales. Universitas Psychologica, 11(1), p. 293-309, 2012. 
VACEK, P.; RYBENSKA, K. Research of interest in ICT education among seniors. Procedia-Social and Behavioral Sciences, 171, p. 1038-1045, 2015.

VIÑALS, A.; CUENCA, J. El rol del docente en la era digital. Revista Interuniversitaria de Formación del Profesorado, 30(2), p. 103-114, 2016.

VIVANCO, M. Muestreo estadístico. Diseño y aplicaciones. Santiago de Chile: Editorial Universitaria, 2005.

Recebido em dia 15 de janeiro de 2019. Aprovado em dia 18 de fevereiro de 2019. 